\title{
FIELD DIET OF THE GRASSHOPPER ABRACRIS DILECTA WALKER (ORTHOPTERA, ACRIDIDAE)
}

\author{
Carlos Frankl Sperber ${ }^{1}$
}

\begin{abstract}
Abracris dilecta Walker, 1870 (Orthoptera, Acrididae, Ommatolampinae) ate leafs of at least 14 plant species, in the families Asteraceae, Lamiaceae, Malvales (Sterculiaceae, Tiliaceae or Malvaceae), Poaceae, Fabaceae, Verbenaceae, Aristolochiaceae, Rubiaceae and Melastomataceae. Elephantopus mollis H.B.K. (Asteraceae) and Hyptis suaveolens Poit. (Lamiaceae) comprised 50\% of the diet. The diet breadth of $A$. dilecta was compared to that of other 11 grasshopper species of the same sub-family, with rarefaction curves. The number of plant species eaten by A. dilecta was greater than that of nine other grasshopper species of the same sub-family (Rhachicreagra spp.) but was lower then two others (Microptylopteryx hebardi Rehn, 1905 and Rhachicreagra astytophallus Jago \& Rowell, 1981). This results are discussed in view of the broad geographical range and possession of developed wings by A. dilecta, which contrasts with most Ommatolampinae grasshoppers.

KEY WORDS. herbivory, diet breadth, tropical dry forest edge, Neotropical region, Brazil
\end{abstract}

Abracris dilecta Walker, 1870 belongs to the Ommatolampinae (Tribe Abracrini), a Neotropical sub-family of the Acrididae. A. dilecta is found in dry, shrubby habitats (ROBERTS \& CARBONELL 1981), while most Ommatolampinae are associated with secondary growth vegetation and dry forest areas (AMÉDÉGNATO \& DESCAMPS 1980; ROWELL 1987), or light-gaps in moist tropical forests (BRAKER 1991; JAGO \& RowELL 1981). A. dilecta differs from most Ommatolampinae for having a broad geographical distribution, from northern Mexico to Argentina, and for the presence of developed wings. These characteristics could lead to a broader diet in A. dilecta than in the species with more restricted distribution and mobility. The aim of this study was to analyse the diet composition (taxonomic range, proportion of host plant species and families in the diet), and breadth (number of plant species ingested) in the field, comparing them with data on other Ommatolampinae grasshoppers.

\section{METHODS}

The field data were collected in an area of $360 \times 30 \mathrm{~m}\left(20^{\circ} 47^{\prime} \mathrm{S}, 49^{\circ} 20^{\prime} \mathrm{W}\right)$, at a border of a semi-deciduous tropical forest, including the pasture beside it, in São José do Rio Preto, State of São Paulo, Brazil. Grasshoppers were captured with translucent plastics vials and kept with moist cotton, fasting for 24 hours, to collect their faeces.

1) Departamento de Biologia Geral, Setor de Ecologia, Universidade Federal de Viçosa. 36570-000 Viçosa, Minas Gerais, Brasil. 
The field diet composition and breadth were determined comparing the faeces of 69 grasshoppers to reference material. To obtain reference faeces material, 51 grasshoppers were kept in the laboratory individually separated, feeding on one of 55 plant species collected in the field (Tab. I). Epithelial reference material was done of the plant species not accepted by these grasshoppers.

The faeces were fixed in a solution of FAA (formalin, acetic acid, and alcohol), macerated over microscope glass plates with glycerine and drawn through optic microscope observations. Every new plant species found in each individual's faeces was recorded as one observation, giving a total of 104 observations. Plant fragment identification was made when possible. When in doubt, plant species in the faeces were lumped, instead of split. The frequency of ingestion was calculated using the number of individuals that presented at least that plant fragment. As several individuals ingested more than one plant species the frequencies did overlap.

The field diet was compared to literature data, with rarefaction curves (KREBS 1989), using the estimates of $95 \%$ confidence intervals. Number of observations were considered as abundance estimates of the plant species ingested in the diet of the grasshoppers. With the rarefaction curves, the estimate of expected number of plant species present in smaller samples than the actual one is possible. This permits comparisons of samples with different size. Only published data on Ommatolampinae diets with details on the frequency of each host plant in the diet could be analysed. The frequency of the host plants in Rhachicreagra spp.'s diet (RowELL 1983a) was estimated multiplying the number of analysed individuals by the minimum estimate of the proportion of the host plant in the diet. In most cases minimum and maximum estimates of host plant frequencies in Rachicreagra's diet did not result in different rarefaction curves. When they did differ, minimum estimates generated rarefaction curves more similar to the rarefaction curve of $A$. dilecta and Microptylopteryx hebardi Rehn, 1905, for which the actual frequencies were known. The diet frequencies of Rhachicreagra spp. overlap, resulting in greater number of observations than individual grasshoppers analysed. $M$. hebardi data (BRAKER 1991) referred to number of individual grasshoppers observed eating in the field. No distinction was done of these frequencies with faeces data.

\section{RESULTS}

Within the plant families eaten, $A$. dilecta ate some but not all of the species present in the field (Tab. I). For example, Orthopappus angustifolius Gleason and Pterocaulon lanatum Kuntze were two common Asteraceae in the field that were not present in the grasshoppers' faeces. Both were ingested by A. dilecta in the laboratory (Tab. I).

A. dilecta presented a diet that included both weeds and forest border species, and among herbs both forbs and Poaceae (Tab. II). A. dilecta ate at least 14 plant species, of at least nine different families. Six plant families ingested by $A$. dilecta are also eaten by other grasshopper species of the same sub-family (GANGWERE \& RONDEROS 1975; Rowell 1978, 1983a,b, 1985; MARQUIS \& BRAKER 1987; BRAKER 1991): Asteraceae (four species ingested), Lamiaceae, Rubiaceae, 
Verbenaceae, Poaceae, and Melastomataceae (Tab. II). There were no prior reports on Ommatolampinae feeding on other three plant taxa: Malvales (Sterculiaceae, Tiliaceae or Malvaceae, not distinguishable through faeces fragments), Fabaceae and Aristolochiaceae.

Table I. Plant species offered to Abracris dilecta grasshoppers to obtain reference faeces material for the identification of the field diet, and abundance of them in the field (+abundant, - rare). Plants not accepted (*) were analysed with epithelium glass slides.

\begin{tabular}{|c|c|}
\hline Plant species & Field abundance \\
\hline Agonandra englerii Hoehne (Opiliaceae) * & - \\
\hline Alibertia cf. sessilis K. Schum. (Rubiaceae)* & + \\
\hline Apocynaceae sp. $1^{*}$ & - \\
\hline Aristolochia esperanze Kuntze (Aristolochiaceae) & + \\
\hline Asclepiadaceae sp. 2 & + \\
\hline Aspidosperma sp. (Apocynaceae)* & - \\
\hline Baccharis dracunculifolia DC.(Asteraceae) & - \\
\hline Bauhinia bongardi Steud. (Caesalpiniaceae) & - \\
\hline Blainvillea cf. biaristata DC.(Asteraceae) & - \\
\hline Byrsonima intermedia A. Juss. (Malpighiaceae) * & - \\
\hline Casearia sylvestris Sw. (Flacourtiaceae) & - \\
\hline Celtis spinosa Ruiz ex. Miq. (Ulmaceae) & + \\
\hline Chaptalia integerrima (Vell.) Burkart (Asteraceae) & - \\
\hline Chomelia sp. (Rubiaceae) & - \\
\hline Cupania cf. oblongifolia Mart. (Sapindaceae) & - \\
\hline Dalechampia tryphylla Lam. (Euphorbiaceae) & + \\
\hline Desmodium adscendens DC. (Fabaceae) & - \\
\hline Desmodium sp. (Fabaceae) & + \\
\hline Diodia teres Walt. (Rubiaceae) & - \\
\hline Elephantopus mollis H.B.K. (Asteraceae) & + \\
\hline Erythroxylum campestre A. St.-Hil. (Erythroxylaceae) * & - \\
\hline Eupatorium sp. (Asteraceae) $)^{\star}$ & - \\
\hline Fridericia speciosa Mart. (Bignoniaceae) $)^{\star}$ & - \\
\hline Guapira sp. (Nyctaginaceae) & - \\
\hline Guettarda cf. uruquensis Cham. \& Schltdl. (Rubiaceae) & - \\
\hline Helicteres ovata Lam. (Sterculiaceae) & + \\
\hline Hyptis suaveolens Poit. (Lamiaceae) & + \\
\hline Lantana camara L. (Verbenaceae) & - \\
\hline Lantana lilacina Desf. (Verbenaceae) & - \\
\hline Lantana sp. (Verbenaceae $)^{\star}$ & - \\
\hline Luehea paniculata Mart. \& Zucc. (Tiliaceae)* & - \\
\hline Malpighiaceae sp. 3 & - \\
\hline Matayba elaeagnoides Radlk. (Sapindaceae) ${ }^{\star}$ & - \\
\hline Miconia albicans Steud. (Melastomataceae) $)^{\star}$ & - \\
\hline Nectandra cf. rigida Nees (Lauraceae) & - \\
\hline
\end{tabular}


Table I. Continued.

\begin{tabular}{lc}
\hline \multicolumn{1}{c}{ Plant species } & Field abundance \\
\hline Orthopappus angustifolius Gleason (Asteraceae) & + \\
Paspalum sp. (Poaceae) & + \\
Peritassa sp. (Celastraceae) & - \\
Pterocaulon lanatum Kuntze (Asteraceae) & + \\
Rhamnidium elaeocarpum Reissek (Rhamnaceae) & + \\
Sebastiania sp. (Euphorbiaceae) & + \\
Serjania communis Cambess. (Sapindaceae) & + \\
Sida cf. glaziovii K. Schum. (Malvaceae) & + \\
Smilax sp. (Liliaceae) & - \\
Solanum paniculatum L. (Solanaceae) & - \\
Terminalia brasiliensis Spreng. (Combretaceae) & - \\
Tournefortia sp. (Boraginaceae) & - \\
Trichilia casaretti C. DC. (Meliaceae) & + \\
Trichilia clausseni C. DC. (Meliaceae) & + \\
Vernonia brasiliana Druce (Asteraceae) & - \\
Vernonia ferruginea Less. (Asteraceae) & + \\
Vernonia ruficoma Schtdl. (Asteraceae)* & - \\
Waltheria indica L. (Sterculiaceae) & - \\
Wissadula cf. subpeltata R.E.Fr. (Malvaceae) & - \\
Xylopia aromatica Baill. (Annonaceae) & - \\
\hline
\end{tabular}

Elephantopus mollis H.B.K. (Asteraceae) and Hyptis suaveolens Poit. (Lamiaceae) were the most frequently ingested plants, followed by one or more of four Malvales species, which could not be separated with the faeces fragments, Poaceae, Baccharis dracunculifolia DC. (Asteraceae), Desmodium sp. (Fabaceae), Vernonia ruficoma Schltdl. (Asteraceae), Lantana camara L. (Verbenaceae), Aristolochia esperanzae Kuntze (Aristolochiaceae), Vernonia ferruginea Less (Asteraceae), Chomelia sp. (Rubiaceae), and Miconia albicans Steud. (Melastomataceae) (Tab. II). Two plant species could not be identified at the family level.

In table III the diet breadth of 19 Ommatolampinae grasshoppers is presented. Of these, 12 could be compared using rarefaction curves (Fig. 1). Microtylopterix hebardi Rehn, 1905 has a more then two fold broader diet then $A$. dilecta, confirmed by the rarefaction curves. Rhachicreagra astytophallus Jago \& Rowell, 1981 and Rhachicreagra brachysphagicerca Jago \& Rowell, 1981 presented a proximate diet breadth with $A$. dilecta, but analysis of the confidence intervals reveals that only $R$. astytophallus has a greater diet breadth then A. dilecta, whereas $R$. brachysphagicerca has a narrower diet breadth. The remaining Rhachicreagra species have all narrower diets than A. dilecta. The diet breadth of Abracris flavolineata (De Geer, 1773) and Leptomerithoprora brevipennis Rehn, 1905 are very similar with $A$. dilecta, but no conclusive analysis was possible because of lack of detailed data on this and other six Ommatolampinae species. 
Table II. Plant species found in the faeces of Abracris dilecta (Orthoptera: Acrididae), number of individual grasshoppers that presented the plant, and plant life form $(\mathrm{H}$ : herb, B: bush, $\mathrm{A}$ : arboreal, $\mathrm{V}$ : vine, $\mathrm{W}$ : weed). Number of grasshoppers analysed $=69$. Weed classification based on BACCHI et al. (1984) and LORENZI (1982).

\begin{tabular}{lcc}
\hline \multicolumn{1}{c}{ Plant species } & Individuals & Life form \\
\hline Elephantopus mollis H.B.K. (Asteraceae) & 31 & $\mathrm{H}, \mathrm{W}$ \\
Hyptis suaveolens (Lamiaceae) & 21 & $\mathrm{H}, \mathrm{W}$ \\
Helicteres ovata Lam. (Sterculiaceae) & & $\mathrm{A}$ \\
Luehea paniculata Mart. \& Zucc. (Tiliaceae) & & $\mathrm{A}$ \\
Waltheria indica L. (Sterculiaceae) & & $\mathrm{H}, \mathrm{W}$ \\
Wissadula cf. subpeltata R.E.Fries (Malvaceae) & 18 & $\mathrm{H}, \mathrm{W}$ \\
Poaceae & 9 & $\mathrm{H}, \mathrm{W}$ \\
Baccharis dracunculifolia DC. (Asteraceae) & 6 & $\mathrm{~B}, \mathrm{~W}$ \\
Desmodium sp. (Fabaceae) & 6 & $\mathrm{H}, \mathrm{W}$ \\
Vernonia ruficoma Schltdl.(Asteraceae) & 3 & $\mathrm{~B}$ \\
Lantana camara L. (Verbenaceae) & 2 & $\mathrm{~B}, \mathrm{~W}$ \\
Aristolochia esperanze Kuntze (Aristolochiaceae) & 1 & $\mathrm{~V}$ \\
Vernonia ferruginea Less. (Asteraceae) & 1 & $\mathrm{~B}, \mathrm{~W}$ \\
Chomelia sp. (Rubiaceae) & 1 & $\mathrm{~A}$ \\
Miconia albicans Steud. (Melastomataceae) & 1 & $\mathrm{~B}$ \\
Not identified species (sp. 1) & 1 & \\
Not identified species (sp. 2) & 3 & \\
\hline
\end{tabular}

Table III. Number of plant species and families eaten by Neotropical grasshoppers of the sub-family Ommatolampinae (Orthoptera, Acrididae) in the field, number of grasshopper individuals analysed $(\mathrm{N})$, and reference. $(\geq)$ At least; $(\leq)$ not more then; (ns) not specified; (1) ROWELL 1983b; (2) RoWELL 1978; (3) BRAKER 1991; (4) ROWELL 1983a; (5) ROWELL 1985.

\begin{tabular}{|c|c|c|c|c|}
\hline Grasshopper species & Plant species & Plant families & $\mathrm{N}$ & Reference \\
\hline Abracris flavolineata (De Geer, 1773) & $\geq 10$ & $\geq 10$ & ns & 1 \\
\hline Abracris dilecta Walker, 1870 & $\geq 14$ & $\geq 6$ & 69 & This work \\
\hline Ateliacris annulicornis (Bruner, 1908) & 1 & 1 & ns & 2 \\
\hline Microtylopteryx hebardi Rehn, 1905 & 52 & 16 & 438 & 3 \\
\hline Microtylopteryx fusiformis Rehn, 1905 & 2 & 2 & ns & 2 \\
\hline Microtylopteryx talamancae Rehn, 1929 & $\geq 4$ & 3 & ns & 2 \\
\hline Leptomerinthoprora brevipennis Rehn, 1905 & $\geq 9$ & $\geq 6$ & ns & 4 \\
\hline Rhachicreagra anchidiphalara Jago \& Rowell, 1981 & 9 & 6 & 39 & 5 \\
\hline Rhachicreagra astytophallus Jago \& Rowell, 1981 & $8-9$ & 6 & 16 & 5 \\
\hline Rhachicreagra brachysphagicerca Jago \& Rowell, 1981 & 7 & 5 & 17 & 5 \\
\hline Rhachicreagra drymocnensis Jago \& Rowell, 1981 & 4 & 4 & 15 & 5 \\
\hline Rhachicreagra gracilis Bruner, 1908 & 3 & 2 & 11 & 5 \\
\hline Rhachicreagra haematodes Jago \& Rowell, 1981 & ns & 1 & ns & 5 \\
\hline Rhachicreagra khayachrosa Jago \& Rowell, 1981 & $\leq 8$ & 1 & 13 & 5 \\
\hline Rhachicreagra melanota Jago \& Rowell, 1981 & 5 & 4 & 15 & 4 \\
\hline Rhachicreagra nothra Rehn, 1905 & $8-10$ & $6-7$ & 46 & 4 \\
\hline Rhachicreagra obisidian Jago \& Rowell, 1981 & 4 & 5 & 16 & 4 \\
\hline Rhachicreagra sp. 215 & 5 & 4 & 15 & 4 \\
\hline Rhachicreagra sp. 243 & 3 & 2 & 12 & 4 \\
\hline
\end{tabular}




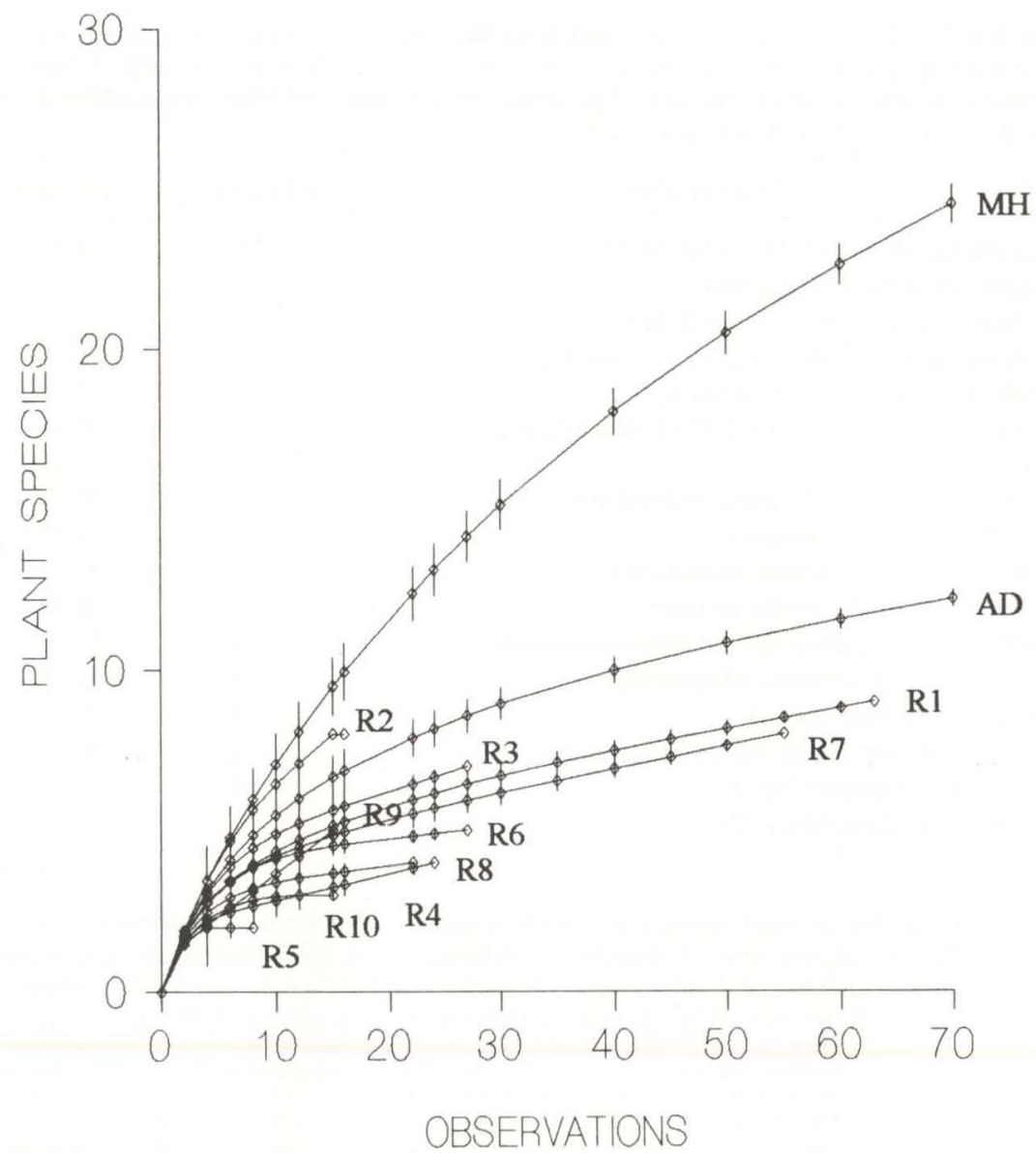

Fig. 1. Rarefaction curves comparing the number of plant species eaten by Abracris dilecta with the diet breadth of 11 other Ommatolampinae grasshoppers. Vertical bars are $95 \%$ confidence intervals. The curves of $\mathrm{AD}$ and $\mathbf{M H}$ are truncated (see Table III). For details on the computation of observation numbers see Methods. (AB) A dilecta, (MH) Microtylopterix hebardi, (R1) Rhachicreagra anchidiphalara, (R2) $R$. astytophallus, (R3) R. brachysphagicerca, (R4) R. drymochnemensis, (R5) $R$. gracilis, (R6) $R$. melanota, (R7) $R$. nothra, (R8) $R$. obsidian, (R9) Rhachicreagra sp. 215, (R10) Rhachicreagra sp. 243. Data for M. hebardi from BAKER (1991) and for Rhachicreagra spp. from RowELL (1985).

Each individual $A$. dilecta had one to four plant species fragments in its faeces (Mean=1.6, Standard Deviation=0.8). This individual diet breadth is the same as that found for Dociostaurus maroccanus (Thunberg, 1815) (Orthoptera: Acrididae: Gomphocerinae) (BEN Halima et al. 1985), but much less than the number of plant species eaten by Taeniopoda eques (Burmeister, 1839) (Orthoptera: Romaleidae: Romaleinae) in a 12-hour period (RAUBENMEIER \& BERNAYS 1993). 


\section{DISCUSSION}

There was no obvious chemical pattern in the diet of $A$. dilecta. The grasshoppers ingested plant species with different alelochemical compositions (e.g., lactone sesquiterpenes in Elephantopus mollis, O. angustifolius and Baccharis dracunculifolia (JAKUPOVIC et al. 1987); alcohol sesquiterpenes in $B$. dracunculifolia (ZDERO et al. 1989); tripterpenoids in Hyptis suaveolens and Lantana camara (PEREDA-MirANDA et al. 1990; QUEIROZ et al. 1990; RAO et al. 1990; SHARMA et al. 1990); coumarins in B. dracunculifolia (ZDERO et al. 1989). In addition, $A$. dilecta did not eat all plants with similar alelochemicals, even if they were abundant in the field. These results agree with observations on other grasshopper species, that also do not present chemical fidelity (BERNAYS \& CHAPMAN 1978; CHAPMAN 1990). This work reinforces, therefore, the thesis that there was no chemical coevolution of grasshoppers and their host plants (BERNAYS \& GRAHAM 1988).

A. dilecta has biological characteristics of generalist species: a broad geographic range, great mobility, and association with secondary vegetation - a possible opportunistic strategy. However it has a narrower diet breadth than two geographically restricted, wingless, grasshoppers: Microtylopterix hebardi Rehn, 1905 (Orthoptera, Acrididae) and Rhachicreagra astytophallus Jago \& Rowell, 1981 (Orthoptera, Acrididae), of the same sub-family. This paradox may have the following reason: wingless grasshoppers must use rare plant species, in unpredictable and ephemeral habitats (forest clearings), favouring diet broadening. Moreover, $A$. dilecta may use more predictable resources than the forest light-gap inhabitants, not due to the vegetation dynamics per se, but due to the ubiquity of certain well-adapted weed species, and the great mobility of $A$. dilecta, which permits searching for specific host plants.

\section{CONCLUSIONS}

The results of this work reinforced the proposal that alelochemicals are of little importance in the determination of grasshoppers diet composition and breadth. The comparison of the diet breadth of A. dilecta with other Ommatolampinae grasshoppers showed an apparent paradox: though $A$. dilecta has biological characteristics of generalist species, it presented a narrower diet breadth than some micropterous, forest light-gap inhabiting grasshoppers. It is proposed that the host plants of $A$. dilecta may be more predictable than light-gap plants, and that the ability of $A$. dilecta to fly may diminish the costs of host plant searching.

ACKNOWLEDGEMENTS. To T. M. Lewinsohn, A. Mesa, F. S. Andrade and M. E. M. Habib, for the suggestions and encouragement. C. S. Carbonell and H. F. Leitão Filho helped with the identifications. Instituto de Biologia, Letras e Ciências Exatas, UNESP, for laboratory and computational facilities. This work was done as part of a M.Sc. dissertation, Departamento de Zoologia, Universidade Estadual de Campinas. Financial support was provided by the CNPq and FMB. 


\section{REFERENCES}

AMÉDÉGNATO, C. \& M. DESCAMPS. 1980. Évolution des populations d'orthoptères d'amazonie du nord-ouest dans les cultures traditionnelles et les formations secondaires d'origine anthropique. Acrida 9 (1): 1-33.

BACCHI, O.; H.F. LEITÃo FILHO \& C. ARANHA. 1984. Plantas invasoras de culturas. Campinas, Instituto Campineiro de Ensino Agrícola, X+906p.

Ben Halima, T., Y. Gillon \& A. LouveauX. 1985. Spécialisation trophique individuelle dans une population de Dosiostaurus maroccanus (Orthopt.: Acrididae). Acta Oecol. (Oecol. General.) 6 (1): 17-24.

BERNAYS, E. A. \& R.F. CHAPMAN. 1978. Plant chemistry and acridoid feeding behaviour, p.99-141. In: J.B. HARBORNE (Ed.). Biochemical aspects of plant and animal coevolution. London, Academic Press, VIII+435p.

BERNAYS, E.A. \& M. GRAHAM. 1988. On the evolution of host specificity in phytophagous arthropods. Ecology 69 (4): 886-892.

BRAKER, E. 1991. Natural history of a neotropical gap-inhabiting grasshopper. Biotropica 23 (1): 41-50.

CHAPMAN, R.F. 1990. Food selection, p.39-72. In: R.F. CHAPMAN \& A. JoERN (Eds). Biology of grasshoppers. New York, John Wiley \& Sons, XII+563p.

GANGWERE, S.K. \& R.A. Ronderos. 1975. A synopsis of food selection in Argentine Acridoidea. Acrida 4 (4): 173-194.

JAGO, N.D. \& C.H.F. RowelL. 1981. Rhachicreagra (Acrididae, Ommatolampinae): forest grasshoppers from Central America with unique aedeagal asymmetry. Syst. Entomol. 6 (2): 179-219.

JAKUPOVIC, J.; Y. JIA; C. ZDERO; V. WARNING; F. BOHLMAN \& S.B. JONES. 1987. Germacranolides from Elephantopus species. Phytochemistry, Oxford, 26 (5): 1467-1469.

KREBS, C.J. 1989. Ecological Methodology. New York, Harper \& Row, Publishers, XII+654p.

LORENZI, H. 1991. Plantas daninhas do Brasil: terrestres, aquáticas, parasitas, tóxicas e medicinais. Nova Odessa, Editora Plantarum, $2^{\text {nd }}$ ed., VIII+ 440p.

MARQUIS, R. \& H.E. BRAKER. 1987. Influence of method of presentation on results of plant-host preference tests with two species of grasshopper. Entomol. Exp. Appl. 40 (1): 59-63.

PeredA-Miranda, R.; P. IBARRA; L. Hernandez \& M. Novelo. 1990. Bioactive constituents from Hyptis species. Planta Med. 56 (6): 560-561.

QueIroz, R.B.; W.H. StUBblebine \& G. SHEPHERD. 1990. Variação na composição de terpenos dentro e entre populações de Hyptis suaveolens (L.) Poit. (Lamiaceae) na região de Campinas -SP. Rev. Bras. Bot. 13 (2): 75-81.

RAO, K.V.R.; L.J.M. RAO \& N.S.P. RAO. 1990. An a-ring contracted triterpenoid from Hyptis suaveolens. Phytochemistry, Oxford, 29 (4): 1326-1329.

RAUBENMEIER, D. \& E.A. BERnAys. 1993. Patterns of feeding in the polyphagous grasshopper Taeniopoda eques: a field study. Anim. Behav. 45 (1): 153-167.

Roberts, H. R. \& C.S. CARBonell. 1981. A revision of the neotropical genus 
Abracris and related genera (Orthoptera, Acrididae, Ommatolampinae). Proc. Acad. Nat. Sci. Phila. 133: 1-14.

RowelL, C.H.F. 1978. Food plant specificity in neotropical rain-forest acridids. Entomol. Exp. Appl. 24 (3): 451-462.

1983a. A revision of the Central American genus Leptomerinthoprora Rehn (Ommatolampinae, Acrididae: Orthoptera). Trans. Amer. Ent. Soc. 109 (2): 179-200.

1983b. Osmilia flavolineata (Chapulín de raya amarilla, Yellow-lined grasshopper), p.750-751. In: D.H. JANZEN (Ed.). Costa Rican Natural History. Chicago, The Chicago University Press, XI+816p.

1985. The feeding biology of a species-rich genus of rainforest grasshoppers (Rhachicreagra: Orthoptera, Acrididae). I. Foodplant use and foodplant acceptance. Oecologia, Heidelberg, 68 (1): 87-98.

1987. The biogeography of Costa Rican acridid grasshoppers, in relation to their putative phylogenetic origins and ecology, p.470-482. In: B.M.

BACCETTI (Ed.). Evolutionary biology of orthopteroid insects. Chichester, Ellis Horwood, 612p.

Sharma, O.P.; R.K. DAWRA \& D. RAmeSh. 1990. A triterpenoid acid, lantadene D from Lantana camara var. aculeata. Phytochemistry, Oxford, 29 (12): 3961-3962.

Zdero, C.; F. Bohlmann; J.C. Solomon; R.M. King \& H. Robinson. 1989. Entclerodanes and other constituents from bolivian Baccharis species. Phytochemistry, Oxford, 28 (2): 531-542.

Recebido em 25.II.1995; aceito em 02.VIII.1996 